\title{
Analytic Bilinear Appearance Subspace Construction for Modeling Image Irradiance Under Natural Illumination and Non-Lambertian Reflectance
}

\author{
Shireen Y. Elhabian ${ }^{1}$ and Aly A. Farag ${ }^{2}$ \\ Computer Vision and Image Processing Laboratory, \\ ECE Dept., University of Louisville, Louisville, KY, 40292, USA. \\ ${ }^{1}$ s.elhabiandfci-cu.edu.eg, ${ }^{2}$ aly.faragelouisville.edu
}

\begin{abstract}
Conventional subspace construction approaches suffer from the need of "large-enough" image ensemble rendering numerical methods intractable. In this paper, we propose an analytic formulation for low-dimensional subspace construction in which shading cues lie while preserving the natural structure of an image sample. Using the frequencyspace representation of the image irradiance equation, the process of finding such subspace is cast as establishing a relation between its principal components and that of a deterministic set of basis functions, termed as irradiance harmonics. Representing images as matrices further lessen the number of parameters to be estimated to define a bilinear projection which maps the image sample to a lowerdimensional bilinear subspace. Results show significant impact on dimensionality reduction with minimal loss of information as well as robustness against noise.
\end{abstract}

\section{Introduction}

Appearance variation due to illumination changes is an inherent challenge in many vision tasks such as recognition. While the lighting function is arbitrary, Belhumeur and Kriegman [1] proved that the set of images of a convex surfaces under distant illumination lies near a low dimensional linear subspace. As such, vision applications, concerned with the recovery of illumination, reflectance or surface geometry from images, e.g. [2], would benefit from low-dimensional generative model which captures appearance variations w.r.t. illumination conditions and surface reflectance properties.

Typically, subspace construction entails performing a dimensionality reduction scheme, e.g. Principal Component Analysis (PCA), on a large set of real or synthesized images of object(s) of interest with different imaging conditions. Despite its great success, the numerical approach has two major problems. First, the acquired/rendered image ensemble should be statistically significant vis-à-vis capturing the full behavior of illumination and reflectance. Second, the curse of dimensionality hinders numerical methods such as Singular Value Decomposition (SVD) which becomes intractable especially with a large number of large-sized realizations in the image ensemble. One way to bypass the need for large image ensembles is to use the harmonic expansion of the image irradiance equation under the convolution framework $[3,4,5]$ to analytically construct appearance subspaces to represent images under fixed pose but different illumination conditions.

In this paper, we propose an approach for analytic bilinear subspace construction to capture the full behavior of appearance variation resulting from non-Lambertian surface reflectance when exposed to complex illumination. We take advantage of the two-fold benefit of the frequency-space representation of the image irradiance equation. First, it decouples the image formation process such that the illumination conditions and surface reflectance characteristics are encoded into the coefficients of a deterministic set of basis functions, which we term irradiance harmonics. This allows the incorporation of prior information about natural illumination and real world surface materials in the subspace construction process. Second and more importantly, the process of finding such subspace can be cast as establishing a relation between its principal components and that of the irradiance harmonics. This resolves the issue of dimensionality since the source of randomness in the imaging process becomes the irradiance harmonics coefficients rather than the whole image realization. Further we take into account the spatial correlation of image pixels while connecting the spatial constraints to the irradiance constraints. This implies applying two linear transforms (hence bilinear) to both the left and right sides of the input image. Results show the superiority of our approach compared to the analytic linear one, e.g. [6,5], in terms of providing significant decrease in subspace dimensionality while maintaining higher approximation accuracy. Potential applications involve constructing generative appearance models which can be used for rendering images under new illumination and in recognition applications. 


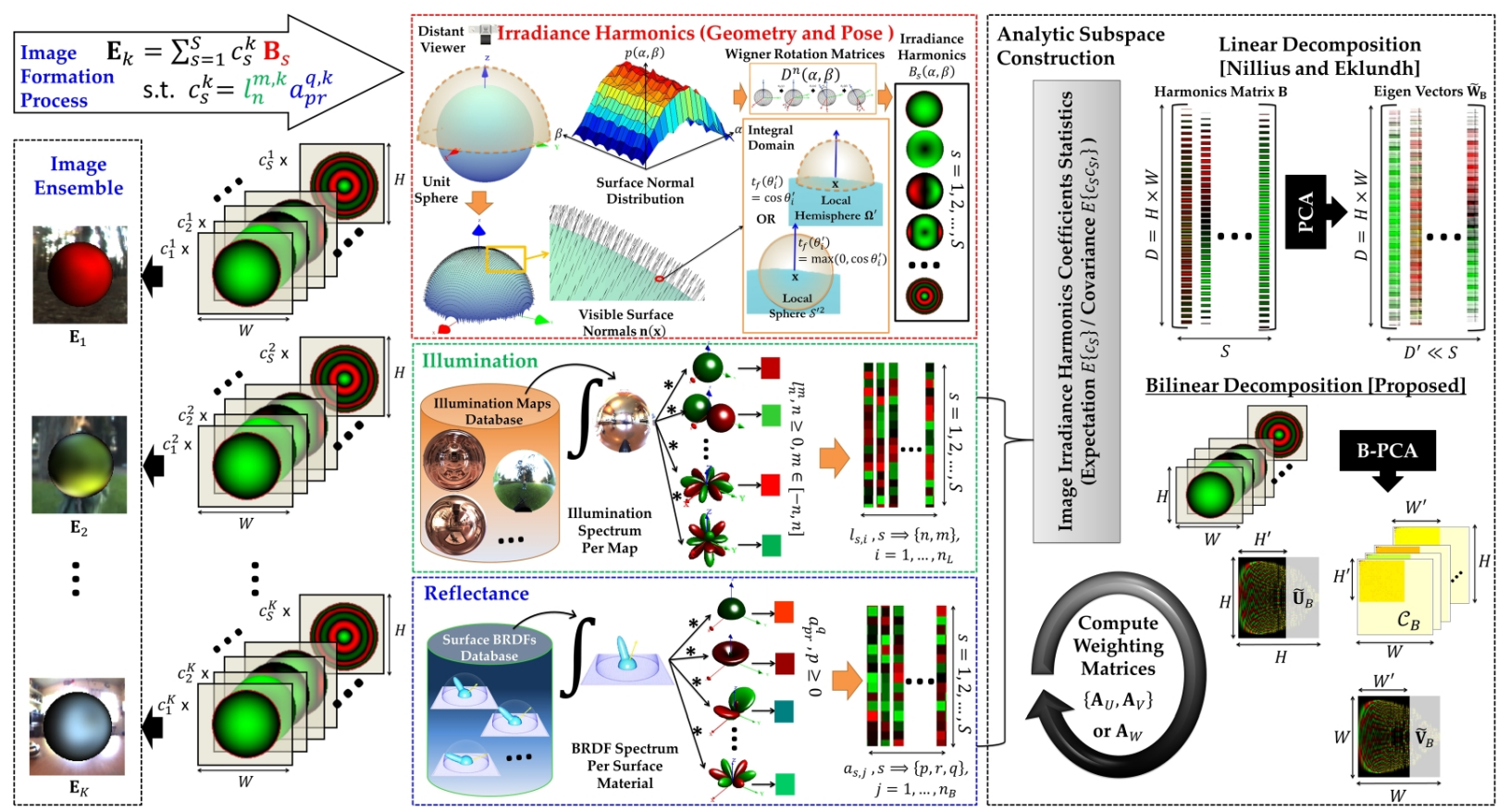

Figure 1. Subspace construction to model illumination and reflectance: assuming fixed pose and surface geometry, the frequency space representation of the image irradiance decouples the image formation process such that the illumination and reflectance are encoded into the coefficients $c_{s}^{k}$ of the irradiance basis functions $\mathbf{B}_{s}$ which are geometry and pose dependent. In case of analytic subspace construction (b), the image coefficients $c_{s}^{k}$ become the random variable instead of the image realization. Thus the inherent curse of dimensionality numerical methods such as Singular Value Decomposition can be handled.

\section{Analytic Bilinear Subspace Construction}

For a specific object geometry under fixed pose, the convolution theory [3] implies a multiplicative framework in the frequency domain where an image is represented as a linear combination of pre-computed basis functions, which we term as irradiance harmonics, $\left\{\mathcal{B}_{s}\right\}$, that are pose and geometry dependent. See Fig. 1. Representing the illumination by its spherical harmonics $(\mathrm{SH})$ coefficients $l_{n}^{m}$ as in $[3,5]$ and the surface reflectance by its coefficients $a_{p r}^{q}$ in the basis of [7], [5] or [8], the image irradiance can be defined as,

$$
E(\alpha, \beta)=\sum_{s} c_{s} \mathcal{B}_{s}(\alpha, \beta)
$$

where $\alpha=\alpha(\mathbf{x})$ and $\beta=\beta(\mathbf{x})$ are the spherical coordinates of the surface normal $\vec{n}(\mathbf{x})$ in the global reference frame, the harmonic coefficients $c_{s}$ encode light and surface bidirectional reflectance distribution function (BRDF) where $c_{s}=l_{n}^{m} a_{p r}^{q}$ with $s$ and its corresponding indices $n, m, p, r$ and $q$ are given by an ordering function of the basis functions [5].

\subsection{Problem Formulation}

Let $\mathbf{E} \in \mathbb{R}^{H \times W}$ be a matrix representation of the image irradiance of the visible surface normals to the viewer such that $H$ denotes height and $W$ denotes width. The objective is to solve for two linear transformations, hence the name bilinear, $\tilde{\mathbf{U}} \in \mathbb{R}^{H \times H^{\prime}}$ and $\tilde{\mathbf{V}} \in \mathbb{R}^{W \times W^{\prime}}$ which map the image space $\mathbb{R}^{H \times W}$ into a lower-dimensional subspace $\mathbb{R}^{H^{\prime} \times W^{\prime}}$ with $H^{\prime} \leq H$ and $W^{\prime} \leq W$ where $^{1}$,

$$
\mathbf{Y} \triangleq \tilde{\mathbf{U}}^{T} \mathbf{E} \tilde{\mathbf{V}}
$$

such that this low-dimensional subspace captures most of the variations observed in the image space due to illumination and reflectance. Thus the objective is maximize the total scatter matrix,

$$
\{\tilde{\mathbf{U}}, \tilde{\mathbf{V}}\}=\underset{\tilde{\mathbf{U}}, \tilde{\mathbf{V}}}{\operatorname{argmax}} \Psi_{\mathbf{Y}} \quad \text { s.t. } \quad \Psi_{\mathbf{Y}}=E\left\{\|\mathbf{Y}-\overline{\mathbf{Y}}\|_{F}^{2}\right\}
$$

where $F$ denotes the matrix Frobenius norm and $\overline{\mathbf{Y}}=$ $E\{\mathbf{Y}\}$ is the origin of the desired subspace. We need to point out that this centering is different from [5](pixelcentering), we opted for image-centering to capture appearance variations which can be used for recognition applications where the contribution of each image is proportional to its deviation from the subspace origin.

\subsection{Analytic Bilinear PCA Derivation}

The optimization problem in Eq. 3 does not admit to a closed form solution allowing solving for the projection matrices simultaneously. Lu et al. [9] used alternating projection method to derive a numerical method for multilinear

\footnotetext{
${ }^{1}$ () denotes lower-dimensional.
} 
PCA for $M$-order tensors. In this work we derive the analytical counterpart for $M=2$ of such an approach.

Let $\mathbf{B}_{s} \in \mathbb{R}^{H \times W}$ be the $s$-th irradiance harmonics of the visible surface normals be represented as a matrix. Hence it can be decomposed into a core matrix $\mathbf{C}_{s}^{B}$ and two orthonormal projection matrices $\tilde{\mathbf{U}}_{B} \in \mathbb{R}^{H \times H^{\prime}}$ and $\tilde{\mathbf{V}}_{B} \in \mathbb{R}^{W \times W^{\prime}}$ such that $\mathbf{B}_{s} \triangleq \tilde{\mathbf{U}}_{B} \mathbf{C}_{s}^{B} \tilde{\mathbf{V}}_{B}^{T}$. The harmonics projection matrices $\left\{\tilde{\mathbf{U}}_{B}, \tilde{\mathbf{V}}_{B}\right\}$ and the core matrices $\mathbf{C}_{s}^{B} \forall s$ are solved for using Bidirectional PCA (BDPCA) [10] in an offline stage using all irradiance harmonics up to illumination and reflectance order $(N$ and $P)$. This step determines the lower dimensions $H^{\prime}$ and $W^{\prime}$ based on the variations inherited from a deterministic ensemble of bases. It is important to emphasis the computational advantage of BD-PCA over PCA where BD-PCA requires $H \times W \times H^{\prime}+H \times H^{\prime} \times W^{\prime}$ multiplications while PCA requires $H \times W \times D^{\prime}$ multiplications for $D^{\prime} \leq H \times W$, $H^{\prime} \leq H$ and $W^{\prime} \leq W$ [10].

As such the image irradiance of the visible surface normals can be rewritten as,

$$
\mathbf{E}=\tilde{\mathbf{U}}_{B}\left(\sum_{s} c_{s} \mathbf{C}_{s}^{B}\right) \tilde{\mathbf{V}}_{B}^{T}
$$

According to the linearity property of the expectation operator, the origin of the image irradiance subspace can be given by,

$$
\overline{\mathbf{Y}}=\tilde{\mathbf{U}}^{T} \tilde{\mathbf{U}}_{B}\left(\sum_{s} E\left\{c_{s}\right\} \mathbf{C}_{s}^{B}\right) \tilde{\mathbf{V}}_{B}^{T} \tilde{\mathbf{V}}
$$

where the harmonics coefficients $c_{s}$ becomes the random variable rather that the image itself.

In order to simplify the process of finding the projection matrices $\{\tilde{\mathbf{U}}, \tilde{\mathbf{V}}\}$, we establish a relation between the orthonormal columns of $\tilde{\mathbf{U}}$ and $\tilde{\mathbf{V}}$ and those of $\tilde{\mathbf{U}}_{B}$ and $\tilde{\mathbf{V}}_{B}$, respectively, such that,

$$
\tilde{\mathbf{U}}^{T}=\mathbf{A}_{U} \tilde{\mathbf{U}}_{B}^{T} \quad, \quad \tilde{\mathbf{V}}^{T}=\mathbf{A}_{V} \tilde{\mathbf{V}}_{B}^{T}
$$

where $\mathbf{A}_{U} \in \mathbb{R}^{H^{\prime} \times H^{\prime}}$ and $\mathbf{A}_{V} \in \mathbb{R}^{W^{\prime} \times W^{\prime}}$ are the weighting matrices encoding such a relation. This renders another benefit of our analytic construction where the optimization problem in Eq. 3 is solved for less number of parameters, i.e. $H^{\prime} \times H^{\prime}+W^{\prime} \times W^{\prime}$ rather than $H \times H^{\prime}+W \times W^{\prime}$.

From Eq. 6 and Eq. 4, the image projection can be simplified to,

$$
\mathbf{Y}=\mathbf{A}_{U}\left(\sum_{s} c_{s} \mathbf{C}_{s}^{B}\right) \mathbf{A}_{V}^{T}
$$

where the solution for $\left\{\mathbf{A}_{U}, \mathbf{A}_{V}\right\}$ matrices is given by the following theorem.

Theorem 1 (Analytic Bilinear PCA) Let $\left\{\mathbf{A}_{U}, \mathbf{A}_{V}\right\}$ be the solution to Eq. 3. Then, based on Alternating Least
Squares, given $\mathbf{A}_{V}$, the weighting matrix $\mathbf{A}_{U}$ consists of the $H^{\prime}$-eigenvectors of $\Upsilon_{V}^{T}$ such that,

$$
\Upsilon_{V}=\sum_{s} \sum_{s^{\prime}} E\left\{\left(c_{s}-\bar{c}_{s}\right)\left(c_{s^{\prime}}-\bar{c}_{s^{\prime}}\right)\right\} \mathbf{C}_{s}^{B} \mathbf{A}_{V}^{T} \mathbf{A}_{V}\left(\mathbf{C}_{s^{\prime}}^{B}\right)^{T}
$$

and given $\mathbf{A}_{U}$, the weighting matrix $\mathbf{A}_{V}$ consists of the $W^{\prime}$ eigenvectors of $\Upsilon_{U}^{T}$ such that,

$$
\Upsilon_{U}=\sum_{s} \sum_{s^{\prime}} E\left\{\left(c_{s}-\bar{c}_{s}\right)\left(c_{s^{\prime}}-\bar{c}_{s^{\prime}}\right)\right\}\left(\mathbf{C}_{s}^{B}\right)^{T} \mathbf{A}_{U}^{T} \mathbf{A}_{U} \mathbf{C}_{s^{\prime}}^{B}
$$

where $E\left\{\left(c_{s}-\bar{c}_{s}\right)\left(c_{s^{\prime}}-\bar{c}_{s^{\prime}}\right)\right\}=E\left\{c_{s} c_{s^{\prime}}\right\}-E\left\{c_{s}\right\} E\left\{c_{s^{\prime}}\right\}$. In the following, we use pseudo (truncated) identity matrices for initializing this iterative procedure where convergence is observed within no more than three iterations.

\subsection{Model-based Bilinear PCA}

The major advantage of the analytic approach is the explicit relation between the principal components spanning the image space and the illumination and reflectance coefficients, allowing for a model-based framework for generic subspace generation. Further, the rotation of the lighting function can be done analytically, reducing the need for acquired data drastically.

Assuming that the lighting function and the surface material/reflectance are independent, one would have [5],

$$
\begin{aligned}
E\left\{c_{s}\right\} & =E\left\{l_{n}^{m}\right\} E\left\{a_{p r}^{q}\right\}, \\
E\left\{c_{s} c_{s^{\prime}}\right\} & =E\left\{l_{n}^{m} l_{n^{\prime}}^{m^{\prime}}\right\} E\left\{a_{p r}^{q} a_{p^{\prime} r^{\prime}}^{q^{\prime}}\right\}
\end{aligned}
$$

where the respective indices are given by the ordering function of the irradiance harmonics functions. Nillius and Eklundh [5] derived a closed form for the expectations and covariances in Eq. 10. As such, databases of $n_{L}$ illumination maps, e.g. [11, 12] and $n_{B}$ real world materials, e.g. [13] can be devised to incorporate prior information in a modelbased framework for analytic subspace construction. See Fig. 1 for illustration.

\section{Connection with Analytic PCA}

Nillius and Eklundh [5] proposed an analytic PCA framework which depends on vector spaces. It can be seen that our proposed solution generalizes the case of vector spaces in [5] where the image is considered as a vector $\mathbf{e} \in \mathbb{R}^{D}$ with $D=H \times W$. The objective is then to find orthonormal projection matrix $\tilde{\mathbf{W}} \in \mathbb{R}^{D \times D^{\prime}}$ which maps the original vector space $\mathbb{R}^{D}$ into a vector subspace $\mathbb{R}^{D^{\prime}}$ with $D^{\prime} \leq D$ where $\mathbf{y}=\tilde{\mathbf{W}}^{T} \mathbf{e}$. Comparing the residual of both cases (linear vs. bilinear), the former case is considered a special case where the column-mode projection matrix is identity such that,

$$
\begin{aligned}
\mathbf{e} & =\tilde{\mathbf{W}} \mathbf{y} \Longleftrightarrow(\tilde{\mathbf{I}} \otimes \tilde{\mathbf{W}}) \mathbf{y}=\mathbf{e} \quad \text { v.s. } \\
\mathbf{E} & =\tilde{\mathbf{U}} \mathbf{Y} \tilde{\mathbf{V}}^{T} \Longleftrightarrow(\tilde{\mathbf{V}} \otimes \tilde{\mathbf{U}}) \mathbf{y}=\mathbf{e}
\end{aligned}
$$


where $\otimes$ is the Kronecker product of two matrices, $\tilde{\mathbf{I}}$ is the identity matrix.

\section{Space and Time Complexity}

Consider a set of $K$-images in the vector space $\left\{\mathbf{e}_{k}\right\}_{k=1}^{K}$. Since each $\mathbf{e}_{k} \in \mathbb{R}^{D}$ is approximated by $\tilde{\mathbf{W}}_{k}$ where $\tilde{\mathbf{W}} \in \mathbb{R}^{D \times D^{\prime}}$ is common for all images, we need to keep $\tilde{\mathbf{W}}$ and $\left\{\mathbf{y}_{k}\right\}_{k=1}^{K}$ for all approximations where $\mathbf{y}_{k} \in$ $\mathbb{R}^{D^{\prime}}$. Hence this requires $D \times D^{\prime}+K \times D^{\prime}=(D+K) \times D^{\prime}$ scalars to store the reduced representation. As such the compression ratio using linear representation is $\frac{K \times D}{(D+K) \times D^{\prime}}$.

In case of bilinear representation, the matrices $\tilde{\mathbf{U}} \in$ $\mathbb{R}^{H \times H^{\prime}}$ and $\tilde{\mathbf{V}} \in \mathbb{R}^{W \times W^{\prime}}$ and $\left\{\mathbf{Y}_{k}\right\}_{k=1}^{K}$ can be used to recover the original image set $\left\{\mathbf{E}_{k}\right\}_{k=1}^{\bar{K}}$ where each $\mathbf{E}_{k} \in \mathbb{R}^{H \times W}$ is approximated by $\tilde{\mathbf{U}} \mathbf{Y}_{k} \tilde{\mathbf{V}}^{T}$. Hence to store the bilinear representation, we need $H \times H^{\prime}+W \times$ $W^{\prime}+K \times\left(H^{\prime} \times W^{\prime}\right)$ scalars with compression ratio $\frac{K \times H \times W}{H \times H^{\prime}+W \times W^{\prime}+K \times\left(H^{\prime} \times W^{\prime}\right)}$, where $H^{\prime}<<D^{\prime}, W^{\prime}<<D^{\prime}$ and $H \times W=D$.

The computation time for the bilinear representation $\mathbf{Y}$ is $\mathcal{O}\left(H^{\prime} \times W\left(H+W^{\prime}\right)\right)$ in comparison to $\mathcal{O}\left(D \times D^{\prime}\right)$ in case of linear representation. Note that our algorithm involves two eigen problems of sizes $H^{\prime} \times H^{\prime}$ and $W^{\prime} \times W^{\prime}$ while the linear counterpart involves an eigen problem of size $D^{\prime} \times D^{\prime}$. Further, the decomposition of irradiance harmonics in the linear case needs to solve an eigen problem of size $D \times D$ while the bilinear case needs two eigen problems with sizes $H \times H$ and $W \times W$. As such, our bilinear representation is computationally more efficient when compared to the linear one.

\section{Experimental Results}

In the sequel, surface reflectance is modeled using the database provided by Mitsubishi Electric Research Laboratories (Merl) [13] which represent a wide variety of surface materials with different diffuse and specular reflection properties $\left(n_{B}=100\right)$. We fit the BRDF measurements up-to reflectance order $P=8$ to (1) spherical harmonics basis [3], (2) hemispherical Zernike-based basis [5] and (3) the isotropic version of hemispherical harmonics (HSH)-based Helmholtz reflectance basis ${ }^{2}$ [8]. The main difference between the three types of basis is modeling the dependency of the surface BRDF w.r.t. the polar coordinates where associated Legendre polynomials is used in (1) while Zernike polynomials and shifted associated Legendre polynomials in (2) and (3), respectively.

We compute the irradiance harmonics for the visible part of a unit sphere, nonetheless, this analysis is applicable to any other geometrical structure. We use illumination order up to $N=10$ and reflectance order up to $P=8$

\footnotetext{
${ }^{2}$ See supplemental material sections 6 and 7 for their closed form.
}

from which we selected $S$-harmonics of the highest average power content where $S$ is chosen such that at least $70 \%$ of the cumulative power content is maintained $(S \approx 580)$. The three types of harmonics ${ }^{3}$ are decomposed using BDPCA [10] to obtain their orthonormal projection matrices spanning the row and column subspaces of the respective harmonics $\left(H^{\prime} \approx W^{\prime}=14\right)$, in addition to their corresponding vector subspaces $\left(D^{\prime}=34\right)$.

In the following set of experiments, we render testing images based on [7] ( $H=W=100)$ using an out-oftraining scenario where the illumination maps from sIBL [12] are used in training the illumination prior $\left(n_{L}=54\right)$ whereas ten illumination maps from [11] are used to render testing images with randomly drawn views. Further, the BRDF that is used to render a testing image is excluded from training the reflectance prior.

Given an image with the same geometry but under unknown natural illumination and reflectance. The trained appearance subspace is fit to such an image using number of principal components which maintain $Q=98 \%$ of harmonics variation. We assess the reconstruction accuracy using the Mean Absolute Error (MAE) between the testing image and the reconstructed one where color values are normalized in the range $[0,1]$.

\subsection{Effect of Irradiance Harmonics}

Fig. 2 shows the average reconstruction errors for each material in the Merl BRDF database based on linear and bilinear subspace construction. It can be noted that bilinear subspace, generally, attains lower error levels for all surface materials when compared to the linear one. This highlights the ability of bilinear representation to encode the intrinsic spatial properties of an image sample compared to the linear one. Further, HSH-based irradiance harmonics (diamond) provides minimal reconstruction error compared to SH-based (circle) and Zernike-based ones (square). This emphasizes the importance of accounting for the physical properties of non-emitting surfaces where a surface point receives incident illumination from the incoming hemisphere oriented by the surface normal at that point. In addition, the spectrum based on HSH-based basis captures more BRDF energy content compared to that of Zernike-based ones. This is based on the fact that at a specific illumination order, the set of associated Legendre polynomials is distinguished by the property that it contains a polynomial for every combination of order and degree, compared to Zernike polynomials which are restricted to even differences between polynomial order and degree.

\subsection{Effect of $Q \%$}

An important factor which affect the subspace dimensionality is the percentage of harmonic variation $Q$ being

\footnotetext{
${ }^{3}$ According to the devised reflectance basis
} 

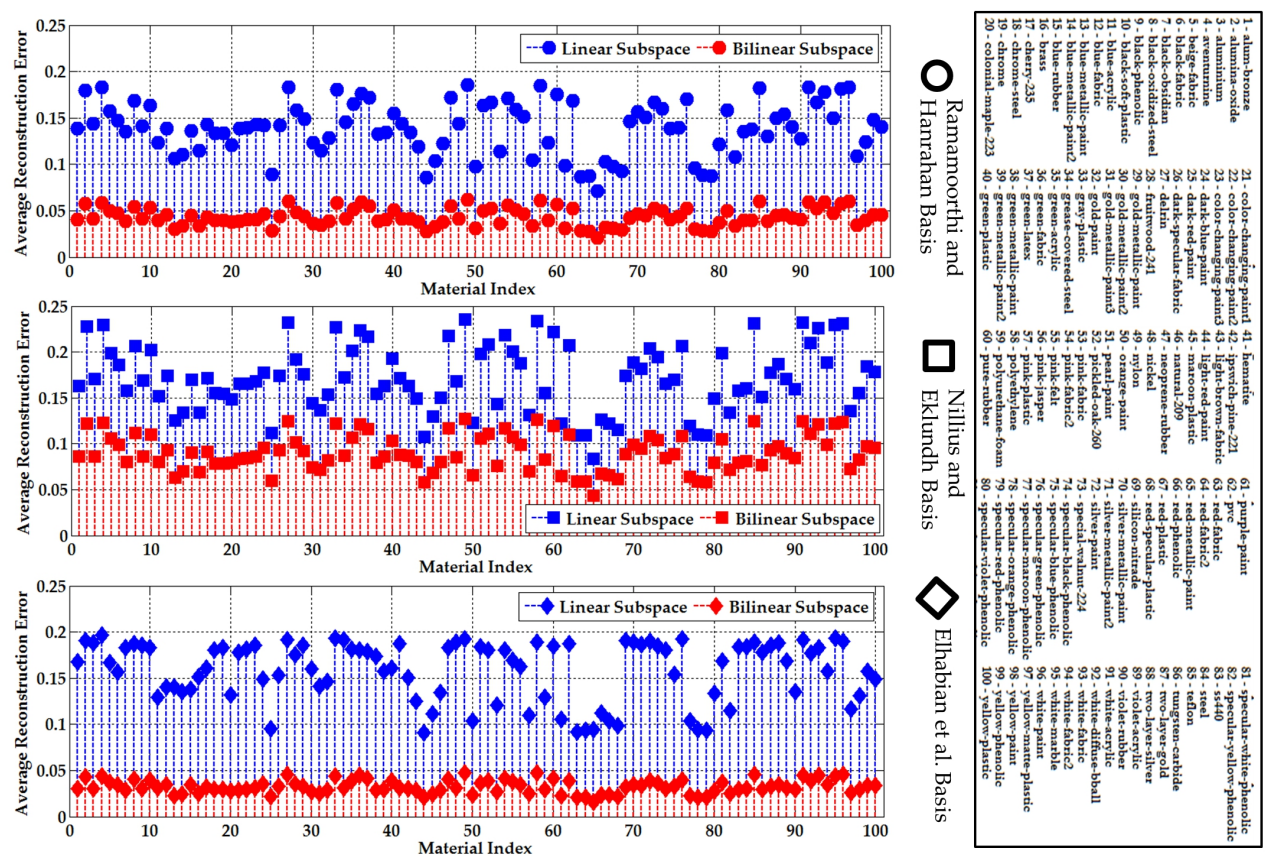

Figure 2. The average reconstruction error for each material in the Merl database based on the 100 rendered testing images per material where subspaces were constructed based spherical and hemispherical irradiance harmonics (refer to the legend) where $Q=98 \%$. Note that bilinear subspaces surpass the linear ones while the Helmholtz HSH-based irradiance harmonics attains minimal reconstruction errors for all the surface materials.

maintained when the irradiance harmonics is decomposed (using PCA or BD-PCA in case of linear or bilinear subspace, respectively). Fig. 3(a,b and c) portrays the average reconstruction error as a function of maintained harmonic variation percentage for linear and bilinear subspaces, respectively, with range $Q \in[85 \%, 99 \%]$. It can be noted that a bilinear subspace can capture the appearance accurately with lower harmonic variation percentage (i.e. lowerdimensional subspace) compared to the linear one regardless of the basis used to model surface reflectance.

\subsection{Effect of Noise}

To test the robustness of the proposed subspace w.r.t. to noise, we added white Gaussian noise with different signalto-noise ratio (SNR) levels to each testing image. To conduct a fair assessment, we have fixed the harmonic variation that each subspace captures such that $Q=98 \%$. Fig. 4(a,b and c) shows the average reconstruction error as a function of SNR levels. One can observe the superiority of bilinear construction in capturing surface appearance even at low SNR levels compared to the linear one. This highlights the benefit of the proposed bilinear appearance model in terms of robustness against noise. Fig. 4(d) shows sample reconstructions for the hippo toy from "Weizmann Photometric Stereo Database" [2] using the pink-fabric BRDF [13] and the Eucalyptus illumination map [11] where our bilinear representation attains minimal reconstruction errors.
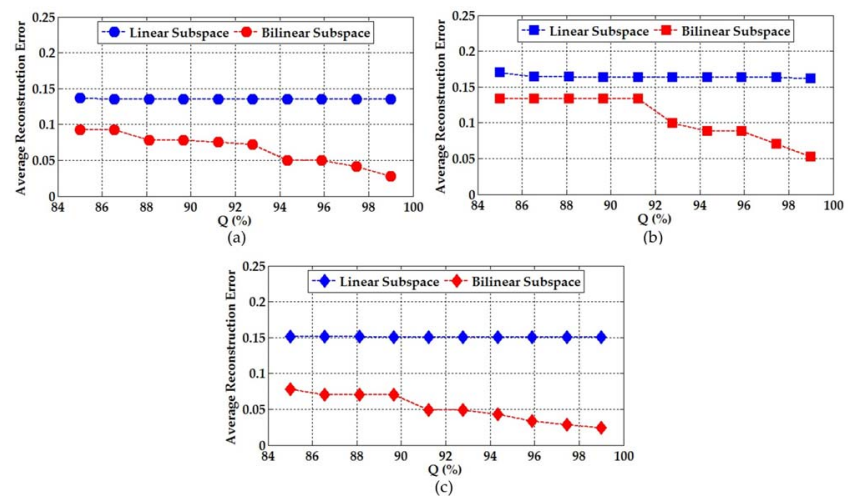

Figure 3. The average reconstruction error as a function of the harmonic variation percentage $(Q \%)$ maintained by the subspace. Surface reflectance is represented using (a) spherical harmonics basis [3], (b) hemispherical Zernike-based basis [5] and (c) the isotropic hemispherical harmonics (HSH)-based Helmholtz reflectance basis [8].

\section{Conclusion}

In this paper, we proposed an analytic formulation for subspace reconstruction to capture the full behavior of complex illumination and non-Lambertian reflectance. Thanks to the frequency-space representation of the image irradiance equation, we were able to incorporate prior information about natural illumination and real world surface materials. The process of finding the analytic subspace was 


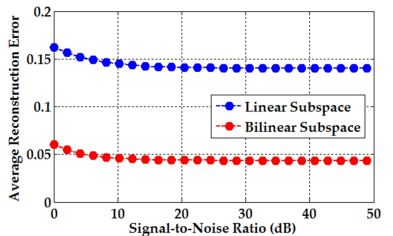

(a)

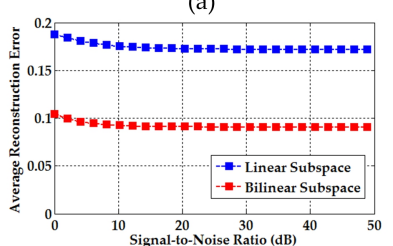

(b)

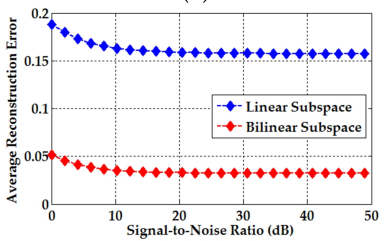

(c)

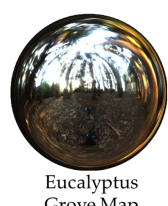

Grove Map
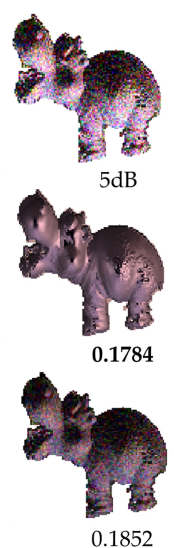

0.1852

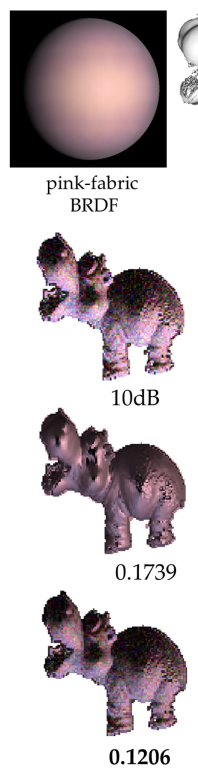

0.1206

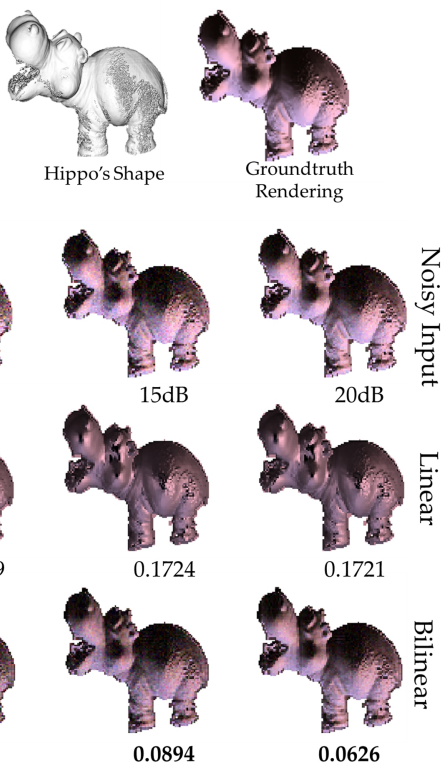

(d)

Figure 4. The average reconstruction error as a function of the signal-to-noise (SNR) ratio in $\mathrm{dB}$. Surface reflectance is represented using (a) spherical harmonics basis [3], (b) hemispherical Zernike-based basis [5] and (c) the isotropic hemispherical harmonics (HSH)-based Helmholtz reflectance basis [8]. One can observe that bilinear subspace still capture appearance even with low SNR levels. (d) Sample reconstructions of the hippo toy from "Weizmann Photometric Stereo Database" [2] using the pink-fabric BRDF [13] under the Eucalyptus Grove illumination map [11] with different SNR levels using irradiance harmonics that are based on Zernike-based hemispherical basis deployed by Nillius ad Eklundh in [5].

cast as establishing a relation between its principal components and that of the irradiance harmonics basis functions to resolve the issue of dimensionality. By representing images as matrices rather than vectors, we were able to lessen the number of parameters to be estimated to define a bilinear projection which maps the image sample to a lowerdimensional bilinear subspace. Despite admitting to an iterative scheme, our approach showed robustness w.r.t. initialization while being able to converge in just one iteration when using truncated full projection. The proposed analytic bilinear PCA showed significant decrease in dimensionality when compared to the linear counterpart while attaining the lowest reconstruction errors. It was further tested against noisy input showing robust image representation even at low SNR levels.

\section{References}

[1] P. Belhumeur and D. Kriegman, "What is the set of images of an object under all possible illumination conditions?," International Journal of Computer Vision, vol. 28, pp. 245-260, July 1998.

[2] R. Basri, D. Jacobs, and I. Kemelmacher, "Photometric stereo with general, unknown lighting," International Journal of Computer Vision, vol. 72, no. 3, pp. 239-257, 2007.

[3] R. Ramamoorthi and P. Hanrahan, "On the relationship between radiance and irradiance: determining the illumination from images of a convex lambertian object," Journal of the Optical Society of America $A$, vol. 18, no. 10, pp. 2448-2459, 2001.

[4] R. Basri and D. Jacobs, "Lambertian reflectance and linear subspaces," IEEE Transactions on Pattern Analysis and Machine Intel- ligence, vol. 25, no. 2, pp. 218-233, Feb 2003.

[5] P. Nillius and J. Eklundh, "Phenomenological eigenfunctions for image irradiance," in Proceedings of the Ninth IEEE International Conference on Computer Vision - Volume 2, ICCV'03, (Washington, DC, USA), pp. 568-575, IEEE Computer Society, 2003.

[6] R. Ramamoorthi, "Analytic pca construction for theoretical analysis of lighting variability in images of a lambertian object," IEEE Transaction of Pattern Analysis and Machine Intelligence, vol. 24, pp. 1322-1333, October 2002.

[7] R. Ramamoorthi and P. Hanrahan, "Frequency space environment map rendering," ACM Transactions on Graphics, vol. 21, pp. 517526, July 2002.

[8] S. Elhabian, H. Rara, and A. Farag, "Towards efficient and compact phenomenological representation of arbitrary bidirectional surface reflectance," in Proceedings of the British Machine Vision Conference (BMVC), pp. 89.1-89.11, BMVA Press, 2011.

[9] H. Lu, K. Plataniotis, and A. Venetsanopoulos, "Mpca: Multilinear principal component analysis of tensor objects," IEEE Transactions on Neural Networks, vol. 19, pp. 18 -39, jan. 2008.

[10] W. Zuo, D. Zhang, and K. Wang, "Bidirectional pca with assembled matrix distance metric for image recognition," Transactions on Systems, Man and Cybernetics - Part B, vol. 36, pp. 863-872, Aug. 2006.

[11] P. Debevec, "Rendering synthetic objects into real scenes: bridging traditional and image-based graphics with global illumination and high dynamic range photography," in Proceedings of the 25th annual conference on Computer graphics and interactive techniques, SIGGRAPH '98, (New York, NY, USA), pp. 189-198, ACM, 1998.

[12] S. I. based Lighting, "http://www.hdrlabs.com/sibl/archive.html."

[13] W. Matusik, H. Pfister, M. Brand, and L. McMillan, "A datadriven reflectance model," ACM Transactions on Graphics, vol. 22, pp. 759-769, July 2003. 